Identité et politique dans la Caraïbe insulaire

\title{
Le droit romain et la servitude aux Antilles
}

Dominique Aimé Mignot

\section{OpenEdition}

Journals

Édition électronique

URL : http://journals.openedition.org/plc/283

DOI : $10.4000 /$ plc. 283

ISSN : 2117-5209

\section{Éditeur}

L'Harmattan

\section{Édition imprimée}

Date de publication : 14 janvier 2004

Pagination : 127-159

ISBN : 2-7475-7061-4

ISSN : 1279-8657

Référence électronique

Dominique Aimé Mignot, "Le droit romain et la servitude aux Antilles», Pouvoirs dans la Caraïbe [En ligne], 14 | 2004, mis en ligne le 03 mars 2011, consulté le 19 avril 2019. URL : http:// journals.openedition.org/plc/283 ; DOI : 10.4000/plc.283 


\title{
LE DROIT ROMAIN ET LA SERVITUDE AUX ANTILLES
}

\author{
par Dominique-Aimé MIGNOT \\ Docteur en droit privé \\ Docteur d'Etat en droit romain \\ Université des Antilles et de la Guyane
}

Le phénomène d'asservissement ou de servitude est caractéristique des sociétés de l'Antiquité : quasiment, aucune n'échappe à cette triste réalité. On peut noter ici et là des assouplissements comme en Egypte ou dans la société hébraïque ${ }^{1}$, voire dans le monde musulman ${ }^{2}$ au temps des Carolingiens. Dans tous les cas, la souplesse et la sûreté du jugement imposent que l'on ne puisse absolument pas opposer facilement les notions de servitude domestique ou douce, d'une part, et d'esclavage rude et brutal, d'autre part ${ }^{3}$. A toute époque, et notamment à l'âge du droit romain classique, les deux conceptions de l'esclavage coexistent. Comme en Grèce, au siècle de Périclès, Rome au $\mathrm{I}^{\text {er }}$ siècle connaît différentes catégories sociales d'esclaves: ceux qui vivent à la campagne $^{4}$, et ceux des villes, ceux qui sont affectés à la rude exploitation des mines ${ }^{5}$ ou qui sont condamnés aux galères, ou aux

\footnotetext{
${ }^{1}$ Encore faut-il considérer les époques et les empires !

${ }^{2}$ Sur la mise en esclavage de nombreux chrétiens à l'époque de Charlemagne : sous la dir. de E. Cavaignac, Le monde musulman et byzantin jusqu'aux Croisades, V. spéc. t. VII (travaux de Gaudefroy-Demombynes, 1931); sur l' "interface » Francs et Arabes, V. le classique ouvr, de Fournier (G.), L'Occident, fin du $V^{\text {ìme }}$ siècle - fin du $L X^{\text {ème }}$ siècle, Paris, A. Colin, 1970, spéc. chap. X, pp. 185-196.

${ }^{3}$ Il ne faut à notre avis pas trop opposer, comme l'a pu faire M. Lengelle, l'esclavage des Anciens (doux) et l'esclavage des « Modernes » (dur). Par exemple une certaine loi Petronia, au début de l'Empire, avait défendu aux maîtres de livrer leurs esclaves aux bêtes sans l'autorisation d'un jugement ! On note de nombreux esclaves dans les mines argentifères et autres Gaulois ou Germains condamnés aux galères...

${ }^{4} \mathrm{Au} \mathrm{II}{ }^{\mathrm{ème}}$ siècle l'Italie est une région de latifundia selon P. Petit, Histoire générale de l'Empire romain, t. I, Le Haut-Empire, p. 149.

${ }^{5}$ Sous l'influence de la doctrine russe, on a restitué la dureté de l'esclavage antique. En ce sens : Kiechle (F,), Slavenarbeit und technischer Fortschrift im römischen Reich, Wiesbaden, 1969; Finley (M.-I.), Slavery in Classical Antiquity, Views and
} 
combats de cirques, et ceux qui, dans de grandes familles nobles, sénatoriales ou équestres, participent à l'activité domestique voire culturelle de leurs maîtres ${ }^{6}$. Les témoignages de la littérature sont nombreux et ne doivent point faire illusion ${ }^{7}$. On se doute bien que la condition d'esclave s'avère plus douce lorsqu'on appartient à la familia urbana de César, à l'instar des affranchis de l'Empereur ${ }^{8}$. Mais la réalité est tout autre pour ceux qui travaillent dans les latifundia de l'Empereur, dans les provinces orientales ou les mines argentifères du Laurion ou dans les villae italiennes 9 . De plus, à ce tableau assez complexe, il convient selon plusieurs sources anciennes de faire état d'esclaves privés et d'esclaves publics, notamment dans les villes d'Asie mineure ou de Pont et Bithynie ${ }^{10}$. On ignore exactement le sort de ces esclaves publics. Mais encore, parmi quelle tranche de population doit-on situer les

Controversies, Cambridge, 1960 (divers articles); Petit(P.) fait le point sur la bibliographie des pays de l'Est, par trop méconnue des Occidentaux: "L'esclavage antique dans l'historiographie soviétique ", in Colloque d'histoire sociale de Besançon, Paris, 1972, pp. 19-27.

${ }^{6} \mathrm{La}$ condition sociale des esclaves attachés à la maison du maître n'a quasiment rien de commun avec ceux qui vivent dans les cultures: V. par ex. les conseils inlassablement répétés de Sénèque dans son De Clementia, I, 18, Ep. 47 ; Cicéron, à propos de la mort de son esclave lecteur dans $A d A t t$. I, 12 ; Pline a également beaucoup de sollicitude à l'égard des esclaves qui vivent sous son toit : notre étude, Droit public et privé d'après les correspondances de Pline le Jeune, th. Et., Lyon, 1989, t. I, pp. 68 et ss.

${ }^{7}$ Cf. Pline le Jeune, Ep. IV : 8 ; VIII : $16 \ldots$ car ses témoignages ne concernent que des représentants de la société sénatoriale ou équestre. Sénèque entend sans doute dénoncer la dominorum saeuitia.

${ }^{8}$ Boulvert (G.), Esclaves et Affranchis impériaux sous le Haut-Empire romain, rôle politique et administratif, Naples, Jovene, 1970 ; Petit (P.), «L'esclavage antique dans l'historiographie soviétique "), in Colloque d'histoire sociale de Besançon, 1970, actes publiés à Paris, 1972, pp. 19-27.

${ }^{9}$ En ce sens, Kouzichtchine (V.-I.), Sur l'histoire de l'économie agraire du II $I^{\text {eme siècle }}$ avant J.-C. au I Ir siècle après J.-C., la pédologie romaine et les sols de l'Italie ancienne (C. r. trad. Du russe en allemand), VDI, 1964, 3, pp. 15-37; idem, Normes et degrés de l'exploitation du travail agricole en ltalie du II $I^{\dot{m} e}$ siècle avant J.-C. au $I^{\text {er }}$ siècle après $J$-C. (en russe dans Antichnoie Obchtchestvo, Moskva, 1967, pp. 37-45).

${ }^{10}$ Halkin (L.), Les esclaves publics chez les Romains, Rome, 1965 ; D.-A. Mignot, "Droit, équité et humanisme d'après la correspondance de Pline le Jeune ", Revue d'histoire du droit français et étranger, vol. 66, 1988, Sirey, spéc. p. 597. 
threptoi, sorte d'enfants abandonnés? La littérature russe, pour ne pas dire soviétique ( $\mathrm{P}$. Petit), a dépeint de façon très pessimiste le sort de ces populations sujettes aux caprices et passions de leurs maîtres.

La condition de l'esclave n'est donc pas si facile à déterminer à Rome. Dans quelle catégorie le situer? Le P. A. Gisler de rappeler de façon lapidaire que le principe du droit romain est que l'esclave est une res. Son statut juridique est celui d'un objet, d'une chose : un « être sujet à propriété et dépourvu de droits $»^{11}$. Cette position n'a guère de sens, à la vérité, en droit romain car la principale distinction n'oppose pas les droits réels mobiliers et immobiliers. C'est d'ailleurs l'erreur de L. SalaMolins que de mettre exagérément cette distinction en avant sur la base d'adages tardifs dont il ne cite pas les références ${ }^{12}$. Bien plus romaine paraît 1'opposition entre les choses dont on dispose, par mancipium ou puissance, de celles qui échappent à notre autorité, les res nec mancipi ${ }^{13}$. L'important pour le Romain consiste à avoir la maîtrise de fait et de droit sur une chose ou sur une personne, esclave ou fils de famille ${ }^{14}$. Etaient donc res mancipi, selon Gaius, les choses qui autrefois étaient les plus précieuses: «les fonds de terre et les maisons situées en Italie, les esclaves et les animaux que l'on a coutume de dompter par le cou ou le dos (obtorto collo dorsove), par exemple les bœufs, les chevaux, les mulets et les ânes... les serviteurs de fonds rustiques, etc. ${ }^{15} \mathrm{Et}$ le même Gaius situe dans son œuvre les esclaves, non parmi les choses, mais

\footnotetext{
${ }^{11}$ Ainsi, l'abbé Gisler reprend-il la position communément admise, sans vérifier quelles furent les véritables tendances du droit romain: L'esclavage aux Antilles françaises, Paris, Karthala, rééd. 1981, p. 31.

${ }^{12}$ Sala-Molins (L.), Le Code Noir ou le calvaire de Canaan, Paris, PUF, $1^{\text {ère }}$ éd. 1987, p. 79 .

${ }^{13}$ Telle est la division fondamentale du droit romain : Cf. Gaius, Institutes, II, 14 a-22 ; Nicosia (G.), Animalia quae collo dorsoue domantur, Iura, XVIII, 1967, pp. 45-107. Le terme mancipia sert même à désigner l'esclave.

${ }^{34}$ Le fils non émancipé se situe sous la puissance du père : il n'est - et les filles aussiqu'un alieni iuris. Sur la question, V. «Filius familias", dans le Nuovo Dizionario giuridico romano, ed. Simone, $1^{\text {ère }}$ éd. 1995, Napoli, p. 210.

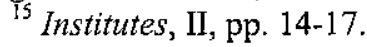


parmi les personnes ${ }^{16}$ car, précise le Pr. Gaudemet, «il lui faut bien reconnaître que ce sont des homines ${ }^{17}$. C'est cette conception, qu'involontairement, les notaires des îles antillaises appliquent dans les inventaires qu'ils dressent lorsqu'ils énumèrent toutes les choses, personnes, biens et animaux qui constituent l'habitation ${ }^{18}$.

On ne reprendra pas ici les terribles répressions qui ont marqué les révoltes ou guerres des esclaves que l'on situe généralement entre 139 et 71 avant J.-C. ${ }^{19}$. Ces conflits firent courir de très graves dangers à l'Urbs. Seule la puissance militaire et l'organisation des légions sauvèrent la République in extremis. La discipline et l'obéissance étaient maintenues par un système de terreur et des châtiments exemplaires dont on a des échos. Le juriste C. Cassius Longinus, membre d'une vieille famille sénatoriale invoque la tradition de sévérité à l'égard des esclaves «car toute modification [de comportement] peut changer le bien en mal » et il faut se protéger des masses dangereuses et d'origines lointaines (Maures, Orientaux, Soudanais, Palestiniens ou Juifs). L'avis de Longinus fut suivi par le Sénat: on révoqua des affranchissements, on appliqua le terrible senatus-consulte silanien et l'on crucifia sur la Via

\footnotetext{
${ }^{16}$ En effet, Gaius parle des esclaves au Livre I (pp. 52-54) de ses Institutes, à propos des personnes, non au Livre II où il traite des choses.

${ }^{17}$ Gaudemet (J.), Inst. Ant., Paris, .Sirey, 1967, p. 549.

18 V. p. ex. parmi les nombreux dénombrements des notaires : L'inventaire de la succession de M. Charles-François Lecointe Bellecour avec Mme. Marie Dieudonnée Moulin, son épouse en date du 18 octobre 1780 (le Sr. Lecointe est capitaine de milice) : après cela figure le nom de l'habitation sise à Baie-Mahaut, paroisse Saint Jean-Baptiste et son activité (sucrière) ; figurent les valeurs : 1) mobilière : $8.710 \mathrm{lv} ., 2$ ) esclaves : 104.200 lv. (36 esclaves), 3) bestiaux : $7.668 \mathrm{lv} .+15.829 \mathrm{lv}$, 4) argent liquide : $34.366 \mathrm{lv}$, 5) mobilier et ustensiles d'habitation: $12.753 \mathrm{lv}$., 6) bâtiments (dont 40 cases à nègres) : $42.034 \mathrm{lv} ., 7$ ) un canal, 8) plantations : 71 cartés de cannes, 9) terres, etc.

${ }^{19}$ La première, de 614 à 621 de Rome (139 à 132 av. n.è.) éclata en Sicile ; la deuxième, de 648 à 652 de Rome (circa 105 à 101 av. n.è.) ; la troisième, de 680 à 682 de Rome (73 à 71 av. J.-C.) eut pour théâtre l'Italie même : on compta 40.000 morts, et plus de 6.000 esclaves furent crucifiés sur les voies menant de Reggio, au sud de l'Italie, vers Rome.
} 
Appia ou condamna aux mines (ad metalla) ou aux bêtes du cirque (ad bestias) un bon nombre de fugitifs ou d'esclaves réfractaires ${ }^{20}$.

Enfin, le droit répressif romain des fautes commises par l'esclave paraît à toute époque d'une particulière dureté. La méfiance à l'égard des esclaves paraît la règle. A la mort du préfet de la ville, Pedanius Secondus, en 61, un débat « terrible » s'engagea au Sénat pour savoir s'il fallait appliquer l'antique coutume de l'extermination de toute la gent servile de la maisonnée du maître assassiné. Il semble que la présomption de complicité ait été retenue, règle déjà admise par le senatus-consulte silanien en l'an 10 de notre ère ${ }^{21}$. Au plan civil, le maître peut abandonner l'esclave qui a commis un délit (abandon dit noxal).

Une historienne russe, E.-M. Schtaerman, a soutenu naguère que la société romaine a connu une véritable crise de l'institution pour des raisons essentiellement économiques ${ }^{22}$. Les esclaves mourraient jeunes ${ }^{23}$

${ }^{20}$ Sénèque affirme dans une de ses Epîtres $(4 ; 8)$ que «l'esclave qui n'a rien à perdre [...] a droit de vie et de mort sur son maître $» ;$ Dictionnaire de Festus, Vo Quot Serui ; Tacite, Annales, XIV : 42, cite des cas d'assassinats de maîtres et du risque d'insécurité dans certaines demeures. C'est alors que le vieil adage repris par Sénèque notamment s'avère d'une triste réalité : tot hostes, tot seruos ! Selon Veyne (P.), « Le seul amour de la liberté n'aurait pas suffit à les pousser à s'enfuir, car un esclave fugitif, loin de se trouver libre comme l'air, se retrouvait sans ressources et n'avait plus qu'à se livrer à un marchand d'esclaves marrons pour se faire vendre, en espérant tomber sur un meilleur maître » (Sénèque, Entretiens, Paris, 1999, p. 1009). P. Veyne cite ici Les bienfaits, IV, 13,1 .

${ }^{21}$ Hermann, "La genèse du senatus-consulte silanien », Revue internationale des droits de l'Antiquité, 1952, spéc. pp. 499-501. Le senatus-consulte néronien semble appliquer le même principe à la familia servile du mari en cas de meurtre de la femme.

22 Sur «la question des esclaves», Schtaerman (E.-M.), «Die Krize der Sklavenhalterordnung im Western des Römischen Reiches ", in Vestrik Drejni Historii (en russe), 1965, t. I, pp. 63-81.

${ }^{23}$ On s'est livré à des « essais » biométriques, mais les résultats sont encore vagues. Ils donnent cependant quelques idées sur l'espérance de vie des esclaves. V. p. ex. : travaux de Szilagyi (J.), comportant de nombreux tableaux sur la durée de vie entre riches et esclaves : «Beiträge sur Statistik des Sterblichkeit in der illyrischen Provinzgruppe und in Norditalien, Gallia Padana », in Acta Arch. Akad. Sc. Hung., 1962, spéc. p. 347. On relève de nombreux cas de mors immatura d'après les inscriptions funéraires : $80 \%$ des esclaves romains mourraient avant trente ans. V. également Henry (L.), «L'âge au 
et surtout ne se renouvelaient pas assez ${ }^{24}$, d'où la nécessité d'asservir d'autres peuples. C'est ce que fera Trajan au début du $\mathrm{II}^{\text {ème }}$ siècle à l'égard des Daces ${ }^{25}$. Cependant, après Hadrien ou Antonin, le limes se resserre ; il faut recourir à d'autres modes de servitude, à la fixation des petits paysans sur la glèbe, à la formation lente de colons que l'on ne pourra plus distraire de leurs terres. Schtaerman a sans doute raison de souligner que les moyens et petits propriétaires, les décurions des villes, souffrent de la diminution du nombre des esclaves et de la médiocrité de leur rendement ${ }^{26}$. On pourra plus loin envisager ce même problème dans d'autres lieux et notamment aux Antilles. Ainsi, apparaît une nouvelle forme d'esclavage dont on veut taire le nom: ce sont les serfs des domaines, des villae ${ }^{27}$, de riches propriétaires... les futurs colons sont attachés à la glèbe.

Toutefois, cette évolution ne doit pas masquer une autre question qui fut non point économique, mais plutôt morale. On a tous à l'esprit les

décès dans les inscriptions funéraires ", in Population, 1959, pp. 327-329 et Ter-VrugtLentz (J.), Mors immatura, Groningue, 1960, spéc. pp. 60-84. Bien évidemment, on retrouve de tels problèmes de surmortalité dans certaines habitations. Parfois ils s'expliquent par des ventes cachées mais il demeure que l'espérance de vie paraît réduite : en ce sens, Sully (L.), Nouvelles tables de mortalité, Paris, PUF, 1969, Travaux et Documents, Cahier n ${ }^{\circ} 53$, p. 161.

${ }^{24}$ Selon Petit (P.), Paix Rom., p. 257, « on peut se demander [parlant des esclaves] s'il faut tenir compte de leur taux de reproduction, et de la rentabilité de leur élevage ». Curieusement, on verra le même problème se dessiner pour les Antilles qui auront pendant tout le XVIII ${ }^{\text {ème }}$ siècle (principalement) recours à la traite, en raison du faible taux de reproduction des femmes en servitude.

${ }^{25}$ Garzetti (A.), Impero romano : l'impero da Tibero agli Antonini (Storia di Roma, t. VI), Bologne, 1959, rééd., pp. 672-674; sur 1'or des Daces : art. de Degrassi dans les Rendiconti dell'Accademia pontificia, 1937; Carcopino(J.), Points de vue sur l'impérialisme romain, chap. II.

${ }^{26}$ Schtaerman, «Die Krize... », pp. 26-36 et 49 et ss. ; V. également Rÿskÿ (I.), «Caractères de la crise de la production esclavagiste dans l'économie agraire de l'Italie au I ${ }^{\text {er }}$ siècle après J.-C. ", in Antichnoie Obchtchestvo, préc., pp. 60-67.

${ }^{27}$ Lot (F.), La fin du monde antique et le début du Moyen-Age, Paris, A. Michel, rééd. 1968, 89 : «Le colonus libre n'a que ses bras et sa famille pour l'aider, point de capital ; et cela nous explique sans doute la facilité avec laquelle il sera lié à la glèbe sous le BasEmpire $»$. 
termes employés par Sénèque, influencé par l'école stoïcienne, à son fidèle ami Lucilius: «J'apprends avec plaisir de ceux qui t'ont rendu visite que tu vis d'une façon toute familiale avec tes esclaves. C'est bien là une attitude digne de quelqu'un d'aussi éclairé et cultivé que toi. Ce sont des esclaves. Non, des êtres humains. Ce sont des esclaves. Non, des camarades logés sous le même toit. Ce sont des esclaves. Non, des compagnons d'esclavage, si l'on songe que la Fortune a sur nous comme sur eux un pouvoir égal ${ }^{28}$.

De même, Pline le Jeune entend reconnaître à ses esclaves domestiques une certaine capacité juridique. Il leur permet même de faire des testaments et il s'engage à les exécuter ${ }^{29}$. Pour Pline, la voluntas compte d'avantage que le scriptum. Il n'est pas le seul à penser ainsi ; on peut y voir un effet de la philosophie stoïcienne ${ }^{30}$. Le droit, après la morale, va lui aussi reconnaître une capacité minimale déjà très remarquée dans l'œuvre du juriste Gaius puis dans celle d'Ulpien ${ }^{31}$.

Elle sera reprise après eux chez d'autres auteurs. Dans cette optique, il est moins question de crise de l'esclavagisme en raison du mauvais rendement bien connu du travail servile; au contraire, c'est plutôt la condition d'esclave qui pose problème. Est-il un bien ? Une res vilis soumise au seul mancipium de son dominus? Ou est-il un des

${ }^{28}$ En fait, selon Veyne (P.), Lucilius maltraitait ses esclaves (Cf. Lettre CVII : 1). Sénèque le réprimande indirectement dans son $E p$. XLVII, Plus loin, Sénèque poursuit sur le même ton: "C'est un esclave! Mais peut-être a-t-il l'âme d'un homme libre ». V. Sénèque, Entretiens, Lettres à Lucilius, Paris, R. Laffont, éd. établie par Veyne (P.), 1993, p. 1094.

${ }^{29}$ Cf. Ep. VIII 16: 1 (in fine) «[...] alternum quod permitto seruis quoque quasi testamenta facere eaque ut lititima custodio », (2.) "Mandant rogantque quod uisum: pareo ut iussus. Diuidunt, foreliquunt, dumtaxat intra domum, nom seruis res publica quaedam et quasi ciuitas domus est $»$.

${ }^{30}$ L'influence de Cicéron et de Sénèque ne fait point de doute sur Ambroise ou SaintAugustin: elle est mise en avant par Schmhilling(O.), Naturrecht und Staat Görresgesellschaft nach der Lehre der alten Kirche, 24, 1914, 247 p.

${ }^{31}$ Ulpien remarque que si l'on se place du côté de l'antique ius civile, l'esclave ne compte pas; à l'inverse, si l'on se situe du point de vue du droit naturel, l'esclave dispose des même droits qu'autrui (V. Dig., 50, 17,32). 
nombreux membres de la domus urbana, une sorte de domestique comme l'affirmaient jadis les moines de Lérins, au Moyen-Age ? Il faut bien reconnaitre que la terminologie devient flottante à la fin du Dominat. C'est que la pensée stoïcienne a rencontré voire conforté sur ce point crucial la philosophie des Pères des Eglises d'Orient et d'Occident ${ }^{32}$. La qualité d'homme est subrepticement reconnue, leur devenir métaphysique est pris en considération, l'humanité s'avère même blessée, mais les faits sociaux sont les plus forts ${ }^{33}$. L'esclave, le colon, le serf d'origine (ou d'ourine) ou de la terre, ou encore sous puissance de son senior (homo de poeste) est bel et bien fixé voire figé : la dépression économique, morale qui débute au III ${ }^{\text {ème }}$ siècle va de pair avec la baisse générale de la culture ${ }^{34}$. Le monde implose et l'Eglise, en Occident, est la seule institution qui ne s'effondre pas: mais elle n'a plus les moyens, dans les siècles d'obscurité, où s'évanouissent les notions d'Etat et d'intérêt public, de remettre en cause l'institution servile ${ }^{35}$. Tout au plus, elle accepte l'oblation des pauvres et miséreux qui désirent être serfs d'Eglise, afin que ceux-ci survivent, eux-mêmes et leurs familles, parce que leur condition réelle sera plus douce et bien plus appréciable que celle des serfs laïques ordinaires. D'ailleurs, nous verrons que la condition des esclaves noirs sur les plantations et domaines ecclésiastiques antillais paraissait objectivement meilleure que celle des

32 Selon Gaudemet (J.), L'Eglise dans l'Empire romain (I $V^{\text {ème }}-V^{\text {ème }}$ siècles), rééd. 1989, p. 566, «Si l'Eglise ne songe pas à condamner l'institution [de l'esclavage], elle s'efforce de remédier au malheur de la condition servile. Derrière la forme juridique, qu'elle tolère, elle retrouve l'homme ». En effet, on peut s'appuyer sur Augustin, Ciu. $D e i$, XIV : 14-15 ; Ambroise, Ep. II, 21. Le concile d'Elvire, par exemple, punit de sept années de pénitence la maîtresse qui a frappé de mort son esclave.

${ }^{33}$ Voir la position du concile de Gangres qui frappe d'anathème ceux qui, sous prétexte de religion, prêchent la révolte servile (dangereuse pour l'ordre public), Cf. canon 3 contre les Eustaniens, Bruns, Canones Apostolorum et Conciliorum saec. IV-VII, 2 vol., Berlin, 1839. Saint-Augustin prône la même doctrine.

${ }^{34}$ Lot (F.), La fin du monde antique et le début du Moyen-Age, Paris, A. Michel, 1968, spéc. chap. 8 .

${ }^{35}$ Sur les classes inférieures à l'époque carolingienne: Perroy (E.), Le monde carolingien, Paris, SEDES, 1974, chap. VII, pp. 163 et ss. ; Fournier (G.), ouvr. préc., p. 88 . 
esclaves de jardin ${ }^{36}$. Ainsi, il en va de même des esclaves en Occident chrétien qui dépendent des différentes coutumes, les unes atroces et sévères, d'autres plus douces ${ }^{37}$. Le Moyen-Age vers l'an Mil hérite cette tare de l'Antiquité qui n'avait connu généralement que des sociétés inégalitaires ${ }^{38}$. La seconde Renaissance de l'Occident fera revivre malheureusement les goûts de l'Antiquité (I.), de là est issue l'application du modèle esclavagiste des Anciens par les Modernes (II.)

\section{LA RESURGENCE DU PHENOMENE ESCLAVAGISTE}

\section{A. UNE POLITIQUE D'EXPANSION COLONIALE}

C'est très curieusement avec le renouveau gréco-romain du monde antique, lors de la seconde Renaissance, qu'apparaissent paradoxalement non seulement le goût pour la beauté et la richesse mais encore celui des découvertes de contrées nouvelles et de la soumission des peuples nouveaux. Dès lors, l'Occident va exporter son trop-plein de forces vives ainsi que ses convictions. Autre paradoxe : alors que le servage paraît dans certaines régions du royaume de France en nette régression, c'est précisément ce modèle que les conquistadores vont imposer aux gens du Nouveau Monde. Le Père Gisler, s'appuyant sur Rinchon, précise que «l'usage de recourir aux esclaves noirs pour la mise en œuvre des territoires du Nouveau Monde eut pour initiateurs les

\footnotetext{
${ }^{36}$ V. p. ex. : Gautier (A.), «Les esclaves de l'habitation Bisdary, 1763-1817», Bulletin de la Société d'Histoire de Guadeloupe, $\mathrm{n}^{\circ} 60,1984$, pp. 13 et ss. : il s'agit d'une ancienne habitation de Jésuites, vendue en 1766 . V. spéc. conclus. où l'auteur prétend que « les esclaves de Bisdary contredisent par bien des aspects les images habituelles sur l'esclavage : beaucoup sont qualifiés, ils sont inclus dans des réseaux de parenté, ils vivent avec leurs père et mère, puis en couple et ont de nombreux enfants [...]; les Jésuites ont mené une politique de mariage originale puisque seuls [en général] $10 \%$ des esclaves guadeloupéens sont mariés ».

${ }^{37}$ On peut toujours se reporter à l'ouvrage classique de Verlinden (Ch.), L'esclavage dans l'Europe médiévale, t. I, Bruges, 1955. Sur l'apparition des esclaves cazés devant plusieurs jours de corvées dès le VI ${ }^{\text {ème }}$ siècle : Fournier (G.), Les Mérovingiens, Paris, PUF, QSJ, 1966, p. 45.

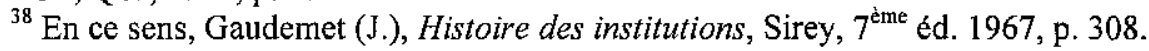


Espagnols et les Portugais ${ }^{39}$ dès le milieu du XVI ${ }^{\text {ème }}$ siècle. Ceci signifie que le système esclavagiste dans toute sa rigueur existait depuis plus d'un siècle ${ }^{40}$ lorsque les colons français s'établirent aux Antilles. Jusque vers les années 1635, la France s'était désintéressée de l'expansion coloniale. Mais une fois le pouvoir du cardinal de Richelieu affermi, ce dernier entend intervenir dans la région des Petites Antilles, sachant bien que la puissance espagnole n'est plus en état de s'y opposer $^{41}$. Nous n'évoquerons pas ici le rôle économique laissé à l'initiative privée et notamment à la Compagnie des îles d'Amérique ${ }^{42}$ dépositaire de la puissance publique. Un fait est certain, l'Etat royal de France ne pose, au milieu du XVI ${ }^{\text {ème }}$ siècle, qu'un regard assez lointain, en raison des guerres civiles dites de religion, regard qui ne sera corrigé

${ }^{39}$ Rinchon, La traite et l'esclavage des Congolais par les Européens, pp. 43-45. V. A. Gisler, L'esclavage aux Antilles françaises (Esc. A. Fr.), rééd. chez Karthala, Paris, 1981, p. 19. La bulle pontificale inter Coeteris (1493) et le Traité de Tordesillas un an plus tard prétendaient attribuer les territoires du sud aux deux puissances ibériques.

${ }^{40}$ D'où la défense des peuples amérindiens par certains religieux. En ce sens : ManhLot (M.), Bartolomé de las Casas et le droit des Indiens, Payot, Paris, 1982, spéc. p. 119 : Les Cortès de Castille s'ouvrent à Valladolid le 4 avril 1542. Les procureurs se sont assemblés au couvent San Pablo: "Nous supplions Votre Majesté de porter remède aux cruautés qui se commettent aux Indes contre les Indiens : ainsy seulement Dieu sera mieux servi et les Indes se conserveront et ne se dépeupleront plus comme elles sont en train de le faire ". Autrement dit, la chambre des Cortès réclame une nouvelle législation.

${ }^{41}$ Sur les desseins du principal ministre de Louis XIII : cf. Lettres patentes du 31 octobre 1626: «L'entreprise a pour but premier de planter la foi chrétienne à la gloire de Dieu et l'honneur du roy [...] et pour condition de mener des prebstres et de cultiver et travailler a toutes sortes de mines et de metaulx». Il n'est pas encore question d'esclavage. Dans le nouveau "contrat » du 12 février 1635, des privilèges sont accordés à la compagnie maritime nouvelle : art. $4:$ «Tous les colons doivent estre catholicques et franscois "; Cochin(V. A.), L'abolition de l'esclavage chez Deshormeaux, L'Harmattan, 1979, p. 246.

${ }^{42}$ Sur l'ordre public social et colonial, V. Cochin (A.), L'abolition..., p. 249 sur le règlement de Tracy, lieutenant-général des isles d'Amérique en date du 19 juin 1664. Ledit règlement veut remettre de l'ordre en matière de mœurs en général (débauche des esclaves et des engagés !) et revenir à une pratique plus chrétienne dans les habitations. Ces mesures marquent indéniablement une réaction à l'époque précédente, à l'ère des Compagnies. 
qu'en 1664, avec la fin du régime des seigneurs propriétaires et la paix intérieure du royaume de France. Pour le reste, on sait par la chronique du P. du Tertre que l'on a envisagé dès les années « 40 » la mise en valeur des îles et notamment de la Guadeloupe par des Noirs importés d'Afrique $^{43}$. Sous l'empire de la Compagnie des Indes occidentales, l'arrivée «en force» des Noirs s'amplifia à tel point que ceux-ci devinrent plus nombreux que les Blancs (y compris les engagés) faisant ainsi basculer lentement les colonies françaises de type de peuplement en colonies dites de plantation.

\section{B. LA CONDITION DE LA MAIN D'GUVRE SERVILE}

Des études solides ont été largement consacrées à la condition des Blancs qui se sont engagés à travailler trente-six mois pour des maîtres avides de rentabilité. Lucien Abenon, à ce sujet, dénonce cette servitude à temps limité ${ }^{44}$. Concurremment, en conséquence de l'échec du travail forcé des Indiens des îles ${ }^{45}$, s'est développé un commerce innommable désigné de trafic triangulaire, à tel point que les premières mesures

${ }^{43}$ Du Tertre (J.-B.), Histoire générale des Antilles, Paris, 1967, p. 1671.

${ }^{44}$ La Guadeloupe de 1671 à 1759, Etude politique, économique et sociale, L'Harmattan, t. II, pp. 72 et ss.; Debien (G.), Les colons des Antilles et leur main d'œuvre à la fin du XVIIlème siècle. Extrait des Annales historiques de la Révolution française, Paris, 1955.

${ }^{45}$ V. Breton (R.), Relations de l'âle de Guadeloupe, t. I, 1656 (éd. Basse-Terre, 1978) : «Aujourd'hui dans l'isle de la Guadeloupe - on l'a deja dict - il n'y a plus d'indiens ; encore qu'ilz viennent ouvent pour comercer. Par contre, on y compte plus de 12.000 franscois, catholicques. Il y a des noirs originaires d'Afrique vendus par leurs roys comme esclaves ou plutost comme betail pour les travaux serviles. Ilz sont a peu pres trois mille de l'ung et l'autre sexe " (Cf. Annexe en latin : De statu et numero Gallorum in insulà: "Coeterum in insula Guadalupa hodie nulli, ut dictum est, morantur indi, etsi ad comercia frequentes ueniant. Gallorum autem catholicum supra duodecim milia numerantur. Nigritarum (qui Africae populi sunt et mancipiorum more et potius bestiarum ad omnia servila opera emuntur a suis regibus; et 1.500 aut 2.000 libris tabaci? Sani et robusti sunt et juvenes ab Anglis aut Hollandis venduntur; gens pia, docilis, fidelis, et uere ad servendium nata, laboriara, parvuo contenta utriusque sexus ad tria et amplius milias, qui omnes fere a nostris in fide instructi et baptisati eo facilus, quo a baptismo daemonibus amplius non uexentur et sapulent ut saepius ante contingebat".) 
relatives à cette « institution » n'émanent que tardivement des autorités locales. Un premier règlement de police date du 19 juin 1664, et outre les peines cruelles coutumières qui y sont confirmées, il prescrit assez curieusement, a priori, aux maîtres de faire baptiser les Noirs et de leur permettre l'assistance aux offices liturgiques, les dimanches et les nombreux jours de fêtes ${ }^{46}$. Sinon, à notre avis, tout est question de faits et non de droit: le régime disciplinaire peut être équilibré chez certains habitants ou atroces chez d'autres. Une espèce de jurisprudence coutumière permet la mise à mort des esclaves fugitifs (les «marrons » de plus de trois mois), ou de ceux qui frappent leurs maîtres et qui doivent être pendus ou étranglés. Ces solutions correspondent d'assez près au sort funeste qui était destiné aux esclaves insoumis ou fugitifs à Rome à la fin de la République ${ }^{47}$. Il faut reconnaître que la justice des nègres en matière de marronage est laissée quasiment à la libre appréciation des maîtres et que cette solution était celle des Romains qui ne sera corrigée que pendant la période classique, voire post-classique du droit romain. C'est grâce au recensement terrier de 1671, ordonné par Colbert, que l'on dispose d'une vision plus exacte de la population des Antilles $^{48}$. La mesure n'est somme toute pas innocente et montre un

\footnotetext{
${ }^{46}$ On peut se reporter à l'ouvr. de Petit (E.), Traité sur le gouvernement des esclaves, (GouvEsc), t. I, 1 et 2.

${ }^{47}$ La règle, sous la Rome archaïque ou royale, est que l'esclave, par nature étranger, ne dispose d'aucun droit : hormis l'hospitalité, tout étranger peut être réduit en esclavage, surtout s'il est prisonnier de guerre. Question controversée en partie par LevyBruhl (H.), «Esquisse d'une théorie sociologique de l'esclavage », art. publié dans Quelques problèmes du très ancien droit romain, Paris, 1934. Sous le Bas-Empire, l'esclave fugitif bénéficie d'une certaine protection : il devient interdit de marquer les esclaves au visage (sans doute parce que l'esclave est lui aussi imago Dei; cf. C. Th., 9, $40,2)$; des constitutions constantiniennes limitent le droit du maître de frapper à mort l'esclave et prohibent certaines tortures, ne pouvant entraîner que la mort, exposant ipso facto les maîtres à être poursuivis pour homicide (Gaudemet (J.), "La législation religieuse de Constantin ", Revue d'histoire de droit étranger et français, 33, 1947, spéc. pp. 38-41).

48 Ainsi le recensement de 1664 (Centre historique des archives nationales, SOM, G1 469) ne cite aucun habitant en Grande-Terre de Guadeloupe; le recensement, avec terier, de 1671 (idem, SOM, G1 468) marque une évolution très nette en habitants et exploitations diverses (indigoteries, sucreries, commerces, etc.)
} 
intérêt marqué - quoique ambigu - de l'Etat royal pour les populations d'outre-mer qui ne cessera de se démentir jusqu'à la fin de l'Ancien Régime $^{49}$.

\section{TRAVAUX PREPARATOIRES ET EDIT DE 1685}

\section{Les prémices}

On aurait tort de croire que la codification de l'esclavage ait été réalisée subitement. Un fait paraît certain : le recensement de 1671 fait état, à côté d'une importante masse servile, d'une catégorie particulière dont la plupart des membres «métissés » ou «mulâtres » sont dits libres de faits ${ }^{50}$. Officiellement le droit applicable aux îles d'Amérique est fixé par l'art. XXXIV de la Charte de la Compagnie des Indes occidentales accordées naguère en 1664. Cet article ordonne aux juges « establiz en tous lieux de juger suivant les loix et ordonnances du royaume [et] de suivre et de se conformer a la coustume des Prevoste et Vicomte de Paris $\rangle^{51}$. En effet, les abus s'étaient multipliés à l'envi dans ces terres lointaines. $\mathrm{Si}$, fait remarquer $\mathrm{A}$. Cochin, le privilège de l'ancienne Compagnie des îles est révoqué le 16 août 1661, c'est qu'elle avait largement dégénéré. Au lieu d'exploiter les terres, elle les vendait; au lieu de civiliser les sauvages, elle les exterminait, et ce, malgré les remontrances des missionnaires ${ }^{52}$. D'où, en conséquence, trois ans plus

\footnotetext{
49 Stehle (G.), «Petit historique des grands recensements antillo-guyanais », BSHG $\mathrm{n}^{\circ} 15,1998$, reprenant en grande partie les données exposées de Satineau pour les populations de Guadeloupe. L'Etat royal ira jusqu'à créer des chambres d'agriculture et une assemblée coloniale en 1787. Il y a là, selon Rovel, déjà une forte idée assimilationniste.

${ }^{50}$ Centre des archives d'outre-mer (CAOM), recens. G1 468. V. les témoignages du P. du Tertre, ouvr. cit., et les arr. du Conseil souv. Guadeloupe, AN, Col. F3 248 (3 décembre 1681). Dessales (P.-R.), Annales du Cons. Souv. de la Martinique, Bergerac, 1786, t. I, pp. 103-104, sur un arr. identique du 16 juillet 1670. Par ailleurs, le Pr. Vonglis, de Martinique, a dressé récemment des notes et commentaires à propos des « arrêts remarquables » de Dessales, rééd. L'Harmattan, 1996.

${ }^{51}$ V. Petit (E.), Droit public ou Gouvernement des colonies françaises (=Gouv. Escl.), Paris, MDCCLXXI, Delalain éd., t. I, pp. 21-34.

${ }^{52}$ En ce sens, Mignot (D.-A.), art. in Bulletin de sociologie et d'histoire de Guadeloupe (BSHG), $3^{\text {éme }}$ trim. 1999, spéc. pp. 42-43.
} 
tard, la création d'une nouvelle Compagnie par l'Edit des 28 mai et 31 juillet 1664.

L'ordonnance du 14 septembre 1672 récidive: elle oblige de nouveau les maîtres à faire instruire leur main d'œuvre et à la faire conduire à la messe tous les dimanches. Un gouverneur menace d'une amende de $2.000 \mathrm{lv}$. de sucre les maîtres récalcitrants et cela, à notre avis, ne visait pas seulement les habitants attachés à la religion prétendue réformée. Surtout, ce texte permet aux religieux missionnaires de tous les ordres de visiter les habitations, d'aller soit dans les jardins, soit ès cazes. Naturellement les commandeurs doivent être catholiques ${ }^{53}$. Là encore il n'est nullement encore question d'organiser le travail servile ni de mettre des bornes à la potestas des maîtres. L'Edit impose aux maîtres ou économies d'envoyer les esclaves et les engagés à la messe; il accorde divers droits seigneuriaux aux associés et proclame in fine que les artisans et sauvages convertis seront « reputez regnicoles et franscois » (art. 35). De façon indirecte, on note encore qu'il est interdit «tant aux negres libres qu'aux sauvages de donner asile aux esclaves, soubz peine, en cas de recidive, d'estre descheux de leurs privilesges de liberte dont ils jouissent $»^{54}$. Enfin, le Mémoire du 13 février 1683 constitue un véritable projet de loi. Il parle déjà de «liberte accordee aulx esclaves » et par ailleurs il affirme que « les affranchis jouiront des privilesges des autres habitans ».

\section{L'Edit de 1685}

L'Edit élaboré par Colbert fils, M. de Seignelay, était destiné aux seules Antilles, puis appliqué ensuite à la Guyane (1704) et l'île Bourbon (la Réunion) avec de légères modifications. Le Code Noir a eu pour fondements directs les Mesmoires de MM. de Blénac, Patoulet et Bégon, respectivement gouverneur général et intendants des îles françaises

${ }^{53}$ En ce sens, P. Labat (N.V.) : «Les commandeurs doivent être impérativement catholiques " (Ord. Du 14 septembre 1672).

${ }^{54} \mathrm{Cf}$. Ordonnances du 12 juillet1678. 
d'Amérique ${ }^{55}$. «Considéré dans son contenu, affirme le P. Gisler ${ }^{56}$, il [1'esclavage] apparait comme une synthèse du droit romain (dont il tient le statut juridique de l'esclave) et des requêtes des moralistes (auxquelles, à des faiblesses près, il est fait droit sous la rubrique des obligations du maître) ». Cet auteur nous entretient donc du modèle gréco-romain dans son maître ouvrage portant sur l'esclavage aux Antilles. Mais cette affirmation doit être à notre avis nuancée : à quel type d'esclavage fait-il réellement allusion? A l'esclavage tel qu'on le perçoit à la fin de la République ? Ou au début de l'Empire romain ? Ou encore à la condition d'esclave aggravée sous le Dominat et à l'époque constantinienne? Voire celle, plus théorique, qui nous est livrée dans les Pandectes de Justinien? Il est difficile, il nous semble même impossible, de définir un type unique et uniforme romain de l'esclavage parce que celui-ci, comme celui qui lui succèdera aux Antilles, fut « évolutif».

Quoi qu'il en soit, le jurislateur de 1685 a eu une certaine vision de l'esclavage antique pour modèle. En conséquence, le servus des îles d'Amérique sera une chose (art. 44: «Declarons les esclaves êtres meubles $[\ldots] \gg)$ à l'instar des autres choses mobilières. Ils pourront donc être vendus, saisis, objets de partage entre héritiers. Un autre article poursuit: «Declarons les esclaves ne pouvoir rien avoyr qui ne soit a leurs maistres, et tout ce qui vient par leur industrie ou par la liberalite d'autres personnes, au autrement a quelque titre que ce soit, être acquis en pleine propriete a leurs maistres [...]» ce qui enlève tout droit de succession entre esclaves. En effet, telle était bien la solution dans le droit romain archaïque issu de l'époque royale. En revanche, l'esclave souffre d'une totale incapacité juridique: il ne peut ni promettre ni s'obliger (art. 28). Il ne peut agir en justice, ni «en deffense ni en demande ». Seuls les maîtres peuvent poursuivre en tant que partie civile et se défendre en matière criminelle en vue de la réparation des outrages et excès commis par ou contre leurs propres esclaves (art. 31). C'est sans doute là une réminiscence très intéressante et très curieuse du damnum

\footnotetext{
${ }^{55}$ En ce sens, Peytraud, Histoire de l'esclavage aux Antilles françaises avant 1789, spéc. pp. 150-158.

${ }^{56}$ Ouvr. préc., p. 26.
} 
iniuria datum et de l'action quod iussu occasionné à un esclave ${ }^{57}$. Les articles 42 et 43 font allusion à la police des esclaves et des maîtres sans aucun doute pour éviter les abus précédents.

Le Code Noir, selon Y. Debbasch, constitue « un système à la romaine; il traduit une indifférence à la macule servile... [mais] en quelques années la structure de type romain a été délaissée, répudiée au profit d'un système purement raciste $~^{58}$. En fait, il faut avoir à l'esprit que l'esclavage à Rome est loin d'être linéaire, qu'il a connu de fortes poussées racistes, et qu'il s'est adouci puis aggravé selon les époques. Le Code Noir reprend la trame esclavagiste romaine dans ce qu'elle fut de pire mais il s'en écarte parfois dans ce qu'elle eut de meilleur ${ }^{59}$. Surtout, il importe d'examiner l'institution dans sa pratique qui, au quotidien, a subi une longue évolution s'écartant fréquemment de la législation de $1685^{60}$.

${ }^{57}$ Le fait de briser un os à un esclave (os fractum servo) était prévu par la Loi des Douze Tables et puni d'une amende de 150 as. Le damnum iniuria datum - ou dommage fait à autrui sans droit - à la chose d'autrui est un délit qui, comme le vol, précise R. Villers, appauvrit la victime, mais qui, à la différence du vol, n'enrichit pas son auteur (ouvr. cit., pp. 250-251). La Lex Aquilia qui date du $V^{\text {ème }}$ siècle av. n.è. est un plébiscite qui dans son chap. III renforce les peines antérieures en matière d'atteinte exercée injustement à l'esclave (ou à l'animal d'autrui).

${ }^{58}$ Debbasch (Y.), Couleur et liberté, le jeu du critère ethnique dans un ordre juridique esclavagiste, Paris, Dalloz, 1967, t. I, pp. 28, 38.

${ }^{59}$ Par ex. un Edit de Claude prive de son droit de propriété le maître qui abandonne son esclave âgé et malade: cf. Suétone, Claudius, XXV, 4 (V. C.J., 7, 1, 3). La Lex Petronia de 19 apr. J.-C. soumit nécessairement au contrôle du magistrat la faculté qu'avait le maître de livrer ses esclaves aux bêtes (V. Dig. 48, 8, 11, 2; 40, 1, 24). Les magistrats romains peuvent même vérifier si les coups donnés en vertu de la dominica potestas correspondent à un motif grave, et même, s'il n'y a pas une sorte de dol du maître à ainsi vouloir se séparer de son esclave : V. Solazzi (S.), L'abuso di diritto in Gai, I, 53, SDHI, XX, 1954, pp. 304-309. On peut considérer la Nov. 14 de Justinien de 535 qui porte sur la tutelle de l'esclave prostituée: V. Sicari (A.), Prostituzione et tutela giuridica della schiava, Un problema di politica ligislativa nell 'impero romano, Bari, Laenccei, 1991, pp. 170 et ss.

${ }^{60}$ Cet aspect semble ne pas avoir été mis en relief par les études récentes. L'art. de Jaubert (P.), «Le Code Noir et le droit romain », in Histoire du droit social, mél. En l'honneur de J. Imbert, publ. sous la dir. de J.-L. Harrouel, Paris, PUF, 1989, 572 p., 


\section{DU MODELE ANCIEN A L'APPLICATION DES MODERNES}

Plusieurs thèmes communs apparaissent si l'on compare le régime romain de l'esclavage tel qu'il s'applique à la fin de la période classique (III ${ }^{\text {eme }}-\mathrm{VI}^{\mathrm{e} m e}$ siècles) et son application tardive sous 1'Ancien Régime. Certains points méritent d'être examinés de près : la capacité juridique de l'esclave, l'existence de familles serviles et du pécule, ainsi que le pouvoir disciplinaire du maître.

\section{A. CaPacite JuRmiQue de L'ESClave}

A l'instar du droit romain classique la capacité de l'esclave est nulle car il a toujours été admis que l'esclavage était une institution relevant du ius gentium par laquelle un homme est soumis à la puissance d'un autre, et ceci, contrairement à la nature.

\section{Le droit d'association et le maintien de l'ordre public}

En principe les esclaves ne doivent point s'unir. Il y va du maintien de l'ordre public et le Code Noir est précis à ce sujet: «Defendons pareillement aux esclaves appartenants a differents maîtres de s'attrouper de jour ou la nuit sous pretexte de noces ou autrement, soit chez l'un de leurs maîtres ou ailleurs, et moins encore dans les grands chemins a peine de fouet ou de la fleur de lys » (art. 16). Dans les faits, on reproche aux libres d'attirer les esclaves et d'avoir avec eux des relations étroites. Les autorités reprochent aux libres de pratiquer le recel de marchandises dérobées ${ }^{61}$. Mais la jurisprudence semble indiquer qu'il

spéc. pp. 321-331 : l'auteur ne voit dans l'esclave qu'une res qui peut devenir une personne si elle est baptisée. En fait, entre la trame du Code et son application au quotidien, il y a loin de la coupe aux lèvres. La flexibilité du droit témoigne souvent d'une application trop rigide mais parfois jugée trop souple, par les Administrateurs, notamment à la fin du XVIII ${ }^{\text {ème }}$ siècle. Il serait intéressant aussi de se reporter au curieux ouvrage de Loisel (A.) fils, Les conférences du Code Noir et du droit romain (BN, Fonds français, 5969) et de le rapprocher du célèbre Gouvernement des esclaves de Petit (E.). Ces œuvres évoquent toutes les différences, malgré l'inspiration du jurislateur de mars 1685, qui existent entre les solutions romaines du droit classique et des dispositions du Code Noir.

${ }^{61}$ En ce sens, Abenon (L.), ouvr. cit., t. I, V. spéc. p. 87. 
y a en ce domaine un profond décalage entre le désir des autorités et la réalité, car les Noirs paraissent tenir des assemblées illicites, et ce fréquemment ${ }^{62}$. Mais il faut admettre que nombreux sont les libres de fait, sorte d'esclaves libres de leurs mouvements dont le statut est indéfinissable ainsi que leur importance numérique ${ }^{63}$. Une ordonnance royale du 10 septembre 1768 rapportée par Moreau de Saint-Mery ${ }^{64}$ interdit la pratique des simples billets, c'est-à-dire de la coutume locale de liberté de fait qui paraît absolument contra legem.

Dès le 6 février 1691, le Code Guadeloupe signale qu'«au préjudice des ordonnances du 6 août 1685, enregistrées en cette Cour et de plusieurs arrests les negres portent journellement de gros bastons ou bengalas [...] ce qui pourroit poser de grands desordres et la mort de quelques uns [...] mesme d'oser lesd. Negres estant pris de boissons soit insulter quelques blancs soit dans les bourgs et lieux eloignes ${ }^{65}$. Un arrêt de fin de siècle (1691) dispose qu' «ayant eu avis que plusieurs particuliers rendent et debitent en detail des eaux de vie de canne chez eux et devant leur porte aux negres en sorte que lesd. Negres s'enivrent et se gâtent de boisson et ensuite se querellent [...] requiert le procureur general que deffense soit faicte auxd. Vendeurs de vendre pour estre bue aucune eau de vie $[\ldots]$ a peine de $300 \mathrm{lv}$. de sucre [...] et de plus grande amende en cas de recidive ${ }^{66}$. La question du maintien de l'ordre public

\footnotetext{
${ }^{62}$ En ce sens, arrêt Conseil Sup. du Cap, 7 avril 1758, art. $1^{\text {er }}$ : «La Cour [...] faict deffense a tous habitants de souffrir les assemblees et ceremonies superstitieuses que certains esclaves ont coustume de fere a la mort de l'un d'entre eulx [...] a peine de 300 lv. d'amende contre les maîtres [...] et de fouet contre les esclaves $»$.

${ }^{63}$ Sur la quest., V. notre étude préc. Il faudrait recenser tous les dénombrements des notaires établis depuis la fin du XVIII ${ }^{\mathrm{ème}}$ siècle. Dans un inventaire dressé pour l'habitation de La Souche - Grande-Terre - on trouve la mention, en 1783, de nombreux esclaves mentionnés comme ne faisant pas partie de la communauté Coudroy-Botté et qui devront rester à La Souche «où ils seront toujours nourris et soignés aux frais et dépenses des propriétaires, sans qu'ils puissent exiger aucuns services ni travaux » [Vanony-Frisch (N.), "Les esclaves de la Guadeloupe à la fin de l'Ancien Régime d'après les sources notariales », $B S H G, 1985, \mathrm{n}^{\circ} 63-64, \mathrm{pp} .80$ et ss.]

${ }^{64}$ Op. cit., vol. V., p. 190.

${ }^{65}$ CHAN, col. F3 221, pp. 803-807. Un arrêt du 30 novembre 1691.

${ }^{66}$ CHAN, col. F3 221, p. 813.
} 
a déterminé, comme à Rome, le législateur à punir les esclaves plus grièvement que les simples sujets $(\mathrm{CN}$, art. 34$)$ en raison du danger qu'ils représentent pour la société coloniale.

\section{De l'institution du pécule}

A la Martinique, les administrateurs rappellent fréquemment les anciennes ordonnances royales ${ }^{67}$ et défendent aux maîtres de traiter financièrement avec leurs esclaves dans le but de les affranchir, en vertu de l'idée que l'affranchissement doit être gratuit et non intéressé. C'est donc que, contrairement aux dispositions du Code Noir, existait une coutume qui permettait une rémunération occulte de certains esclaves de par leurs activités. Nous retrouvons là l'existence du pécule romain fruit de l'activité de l'esclave ${ }^{68}$. Et les témoignages paraissent assez nombreux sur ce point ${ }^{69}$. C'est le cas pour Marie-Jeanne dont le maître est le Sr. Belost, maître en chirurgie au bourg de Saint-François de BasseTerre. Marie-Jeanne est mulâtresse de dix-neuf ans, elle est estimée à $3.300 \mathrm{lv}$., "marchande, bon sujet, tient une boutique de faïence et d'alimentation: elle est portée pour mesmoire a affranchir $\gg{ }^{70}$. Une certaine Thérèse, sujet âgé, est déclarée libre ${ }^{71}$. Elle restera sur

${ }^{67}$ Ord. Des 15 juin 1736 et $1^{\text {er }}$ février 1766.

${ }^{68}$ V. Chauleau (L.), Recherches sur l'histoire sociale de la Martinique (1635-1715), th. Ec. Chartes, 1961 (dactyl.), V. spéc. t. I et II sur les affranchis morts $a b$ intestat, pp. 321 et ss.

${ }^{69} \mathrm{P}$, ex. pour Saint-Domingue, il a été donné 845 libertés dont 203 gratis et 108 pour un prix modéré. Parmi les libres de savane on note des esclaves attachés à l'habitation mais qui ne figurent plus officiellement sur les listes notariales de dénombrement. En ce sens, Vanony-Frisch (N.), "Les esclaves de la Guadeloupe... ", BSHG, $\mathrm{n}^{\circ}$ 63-64, 1985 : Sur l'habitation Bisdary les malades sont soignés par un chirurgien, l'année 1768 ; on peut lire ailleurs que « le negre Crisostome, agé de 14 ans, est actuellement a la petite-case, chez le Sr. Bioche, chirurgien, pour raison d'ulcère déclaré lors de l'inventaire» [Gautier (A.), op. cit., p. 25].

${ }^{70}$ Cf. Inv. Mimerel, acte $2 / 195,7$ décembre 1775 . De telles femmes selon VanonyFrisch « exercent leurs activités au bourg, hors de la maison du maître, dans une semiliberté ».

${ }^{71}$ Debien (G.), Les esclaves aux Antilles françaises XVII ème $X V I I I^{\text {eme }}$ siècles, BasseTerre (Guadeloupe), Société d'histoire de la Guadeloupe, 1974, p. 386, citant une missive du 11 avril 1768 . 
l'habitation. La missive poursuit ainsi: «Elle donne pour prix de sa liberté les $405 \mathrm{lv}$. que j'avois a elle, une vieille cavale qui peut valoir de 90 à $100 \mathrm{lv}$. et un billet de $100 \mathrm{lv}$. sur un negre libre ». On note aussi des libertés de savane pour des ouvrières d'habitation, des lingères ou cuisinières. Une certaine Zabelle, esclave de l'indigoterie Sainton, au Fonds-des-Blancs (Saint-Domingue), est présentée comme «absente de l'habitation et maîtresse de ses volontes en consideration de ses services $1{ }^{72}$. Ailleurs, un certain Eube perçoit des sommes régulières en septembre 1786 et en juillet 1788 et dans les documents il est dit tantôt «negre d'habitation », tantôt «libre de l'habitation », tantôt « libre " ${ }^{73}$. Ces femmes mettaient de longues années pour accumuler ce qu'il faut bien appeler un pécule. Ces opérations d'affranchissement s'effectuaient par degrés successifs, ce qui était très mal vu de l'administration qui ne percevait pas la taxe d'affranchissement ${ }^{74}$. Ce faisant, la pratique coutumière du pécule aux Antilles n'était qu'une lointaine et timide mais remarquable - réminiscence du pécule romain ${ }^{75}$. Elle correspondait à d'antiques usages car, pour rentabiliser l'achat des esclaves, dès 1660 , on leur apprend «toutes sortes de metiers en quoy ilz sont fort adroicts, hommes et femmes ${ }^{76}$. J. Fallope résume la question du pécule en écrivant que «la loi fondamentale de l'esclavage stipule (sic) que

${ }^{72}$ Cf. Invent. des biens de J. Sainton, 29 janvier 1787 (CHAN, SOM, notariat de SaintDomingue).

${ }^{73}$ En ce sens, Debien (G.), «Les esclaves... », SHG, 1974, p. 383.

${ }^{74} \mathrm{La}$ taxe d'affranchissement a été très sujette à caution et vivement critiquée. Finalement une ordonnance royale du $1^{\mathrm{er}}$ février 1766 (art. 27) la supprima, car on veut revenir au principe de la gratuité de l'affranchissement prévue au $C N$.

${ }^{75}$ Sur la notion de pécule, V. Ourliac (P.) et de Malafosse (J.), t. III, Le droit familial, p. 44 : « La plupart des actes de la vie économique ont un caractère synallagmatique, ils comportent des obligations réciproques. Le fils [de famille] se trouve dans la même situation que l'esclave qui peut servir à son maître d'instrument d'acquisition. C'est là une application de la théorie de l'accession. Le fils, comme l'esclave, est un élément du patrimoine appartenant au pater; dès lors, les acquisitions provenant de cet élément accroissent le patrimoine du pater $\gg$. Le préteur, en conséquence, permettra aux créanciers de l'esclave (ou du fils, sujet alieni iuris) d'agir contre le père ou le maître (dominus) qui a concédé le pécule parfois qualifié de «profectice ». Le magistrat accorde alors une actio adiecticiae qualitatis, ou action avec transposition de noms.

${ }^{76} \mathrm{CHAN}, \mathrm{col}$. C8, B1, F7. 
l'esclave ne peut posséder: l'art. 28 du Code Noir concerne cette défense. Cependant, pendant toute la durée de l'esclavage, des esclaves sont arrivés avec ou sans l'assentiment du maître à accumuler un petit pécule ${ }^{77}$.

D'ailleurs, il convient de remarquer que, tardivement et officiellement, l'idée du pécule a fait son chemin au début du $\mathrm{XIX}^{\text {eme }}$ siècle. Selon J.-M. Breton, «il faudra attendre une loi de 1845 pour voir progressivement réduites les peines corporelles et permettre enfin à l'esclave de monnayer sa liberté, en lui garantissant la propriété d'un pécule qu'il pourra utiliser s'il entend acquitter le prix de sa rançon $»^{78}$. Les faits démontrent qu'une telle pratique, d'essence romaine, existait en fait incontestablement dès le milieu du $\mathrm{XVIII}^{\text {ème }}$ siècle.

\section{B. LA FAMILLE SERVILE}

\section{Notion de famille}

Si l'on se reporte au Dictionnaire de Furetière (1690) le mot «famille se prend pour un mesnage compose d'un chef, de ses domestiques, soit femmes, enfans ou serviteurs ». De même, du Tertre inclut les esclaves dans la familia du maitre, à la mode romaine " avecq son epouse, ses enfans et les serviteurs blancs ». Selon le Jésuite Pelleprat $^{79}$, "l'esclave trompe par sa femme» peut demander à son maître une autre femme ${ }^{80}$. Il résulte bien de tous ces témoignages que le terme famille est pris ici au sens large et qu'il conserve son sens latin. On peut même parler très précisément de la famille rustique (familia rustica) traditionnellement opposée à Rome à la familia urbana. Les esclaves des campagnes, aux Antilles, comme dans le monde romain ont une situation

\footnotetext{
${ }^{77} \mathrm{~V}$. « Résistance d'esclaves et ajustement au système : le cas de la Guadeloupe dans la première moitié du XIX ${ }^{\text {ème }}$ siècle », $B S H G, \mathrm{n}^{\circ} 67-68$, pp. 31-52, spéc. p. 44.

${ }^{78}$ C.r. trimestriels des séances de l'Académie des sciences d'outre-mer, t. LVIII, 1998, pp. 165-187.

${ }^{79} \mathrm{Cf}$. Relations des missions des RR PP de la Compagnie de Jésus..., Paris, 1655.

${ }^{80} \mathrm{Y}$ a-t-il là un témoignage de l'existence d'un contubernium placé sous le contrôle du maître? Les études font défaut en ce domaine.
} 
plus rigoureuse et plus sévère : à l'inverse, les esclaves des villes ou des bourgs plus proches de leurs maitres, et où les mœurs sont moins rudes, sont moins mal traités ${ }^{81}$. Un certain nombre d'esclaves suivent leurs maîtres en métropole et leur retour aux Antilles pose de nombreux problèmes au législateur qui craint la propagation des idées nouvelles ${ }^{82}$.

\section{Mariage et servitude}

Un constat: la gent servile se renouvelle peu dans les Antilles françaises. Il y a pour expliquer cette situation, assez dramatique du point de vue des habitants, plusieurs raisons. Il semble que la première soit toute simple : la femme africaine n'a guère envie de donner naissance à une progéniture subissant le joug de la servitude. Par ailleurs la pratique du mariage entre esclaves paraît quantitativement faible: les liaisons

${ }^{81}$ La situation au début du XIX ${ }^{\text {ème }}$ siècle est héritée des usages et pratiques du XVIII ${ }^{\text {ème }}$. Pour le $\mathrm{XIX}^{\text {ème }}$ siècle, cf. Adélaïde-Merlande, «Problématique d'une histoire de $l^{\prime}$ esclavage urbain, Guadeloupe, Guyane, Martinique vers 1815-1848 ",BSHG, ${ }^{\circ} 65$, pp. 3-23, spéc. pp. 20 et ss. Une confrérie des esclaves, instaurée en 1752 par les Jésuites en Guadeloupe, ne laisse pas de nous interroger : sur la quest., Abenon (L.-R.), ouvr. cit., t. II, p. 59 Il conviendrait de faire une étude sur les nourrices, cuisiniers, cochers, femmes de chambre, sages-femmes, valets, charpentiers, tonneliers, souvent créoles, et qui sont fréquemment affranchis alors que les « esclaves de terre " (Debien, op. cit., p. 376) sont ignorés des économes et des maîtres.

${ }^{82}$ En ce domaine, les travaux sont assez nombreux. Déjà au XVIII ${ }^{\text {eme }}$ siècle, Poquet de Livonnière cerne le problème dans ses Regles de droit françois, et nous entretient des « esclaves venant de noz isles d'Amerique $[\ldots]$ qui viennent avec leurs metres $[\ldots]$ et qu'on a dessein dy ramener » et Durand de Maillane d'ajouter que les nègres " venant dans le royaume a la suite de leurs maîtres sont censés ne jamais quitter leur paiis ny joug de la servitude ». Le fait de fouler la terre de France ne peut affranchir que des esclaves étrangers, ce qui est contraire à la règle originaire appliquée au XVII ${ }^{\text {̀me }}$ siècle et au début du XVIII ${ }^{\text {eme }}$ (CHAN, col. C8 A7, missive de Dumaitz au Secr. d'Et., 2 juillet 1682, cf. Edit d'octobre 1716, art. 15). Elisabeth (L.), dans un mémoire de DES sur Les gens de couleur à Bordeaux au XVIII ${ }^{\text {eme }}$ siècle (1960) dépeint le décalage social lorsque les Noirs rentrent dans les îles. Dès lors, on contrôle tous les départs et les arrivées des gens de couleur (cf. arr. Cons. Et., 11 janvier 1778 et Ord. De 1778, sur la durée des séjours en France de ces populations). L'étude ancienne de Vignols (L.) sur "Les esclaves coloniaux en France aux XVII ${ }^{\text {eme }}$ et XVIII ${ }^{\text {eme }}$ siècles et leur retour aux Antilles », in Mél. bretons et celtiques offerts à L. Loth, Paris, 1927, fait état de la police des Noirs dans les villes de l'ouest et à Paris. 
ressemblent plus au contubernium décrit par les textes romains ${ }^{83} \mathrm{qu}$ 'au mariage sacrement de l'Eglise malgré les articles 10 et 11: «Les solempnites prescrites par l'ordonnance de Blois et par la Déclaration de 1639 , pour les mariages, seront observees, tant a l'egard des personnes libres que des esclaves, sans neantmoins que le consentemen du pere et de la mere de l'esclave y soit necessaire, mais celuy du maistre seulement $^{84}$. [...] Deffendons aussy aux maistres d'user d'aucunes contraintes sur leurs esclaves pour les marier contre leur gré ». Il y a là un effet du droit consensualiste de l'Eglise (l'échange des verba de praesenti) qui doit être libre, l'accord du maitre étant requis en vertu de sa seule auctoritas.

Dans un second temps, lorsque la population servile se créolise, il semble que les raisons qui expliquent l'insuffisance de procréation soient différentes. Un curieux texte nous éclaire à ce sujet : « Sa Majeste a este informee de l'extraordinaire prostitution qui regne parmi les negresses et du peu de soin qu'on a eu jusqu'à present de l'empescher et comme Elle [désire] que ce desordre soit reprime, non seulement pour l'interest des bonnes mœurs et de la religion, mais aussi pour celuy de la colonie par ce que cette prostitution empeche les femmes de devenir grosses et qu'elle tireroit des negres qui naitroient dans le paiis. Sa Majeste veult qu'elles

\footnotetext{
${ }^{83}$ En cas de simple contubernium, les enfants sont naturellement esclaves et tiennent de la condition de la mère. Mais se pose la vieille question du mariage entre une libre et un serf, notamment parmi les esclaves impériaux : là encore, la tare servile demeure et les enfants nés d'une telle union sont illégitimes (CIL, VI, 15114; 29513, etc.) En revanche, les esclaves peuvent s'unir librement, entre frères et sœurs, oncle et nièce, puisque n'existe aucun empêchement dirimant. Toutefois, on note des unions serviles à Rome qui furent durables (CIL, VI, 8676: vie commune pendant quarante ans sine qu(erala). Sous l'empire de certaines législations, on apprécie la qualité de la mère au début ou pendant la grossesse pour faire jouir l'enfant de la liberté (cf. Gaius, Instit., 1, 82 ; Castello (V.-C.), La condizione del concepto da libero e schiava et da libera $e$ schiavo, St. Solazzi (1948), pp. 232-250. Le CN confie la légitimité aux enfants nés d'un maître et d'une esclave, par effet de mariage subséquent (= sub pallio).

${ }^{84}$ En ce sens : décrétale d'Adrien IV ; contra : décrétale de Grégoire IX, Liv. IV, Tit. 9, de conjugo servorum.
} 
s'appliquent a faire les reglemens necessaires et qu'elles portent autant que faire se peut les negres et les negresses a se marier entre eux $»^{85}$.

C'est d'ailleurs en ce sens qu'il faut comprendre 1'attitude de certains religieux aux Antilles. Par exemple le Jésuite Mangin fait état à Saint-Christophe de conduites apparemment curieuses: "Quelques mestres $[\ldots]$ sont alles a ces exces [...] que, n'ayant que des negresses, ilz ont paye des negres estrangers comme des etalons pour en avoyr les enfans qui sont toujours au maistre de la mere $»^{86}$. Solution normale puisqu'elle existait déjà en droit romain classique en raison de la seule parenté cognative en application de l'adage connu partus ventrem sequitur ${ }^{87}$. Mais cette pratique semble assez minoritaire et l'on ne peut guère parler d'existence de haras humains dans les îles du Vent. Il y a un pourcentage d'ateliers important «sans hommes » ou presque. En 1664, au commencement de l'importation massive de Noirs d'Afrique, 22,6\% des ateliers martiniquais sont féminins et on dispose du même nombre pour l'année 1680 selon les dénombrements : le phénomène est à peu près semblable en Guadeloupe pour l'année $1664(22,4 \%$ des ateliers sont composés de femmes).

En conséquence, compte tenu du peu de femmes blanches, et de l'échec de l'importation des paysannes auvergnates ou des régions de

\footnotetext{
${ }^{85}$ CHAN, F221 F607, missive de Begon en date du 22 avril 1684.

${ }^{86} \mathrm{Cf}$. Mangin, Bibl. de Carcassonne, VII, p. 112.

${ }^{87}$ Sur la quest. : Kaser (M.), Partus ancillae, ZSS, LXXV (1958), pp. 156-200. La difficulté provient des mariages mixtes, aussi bien à Rome, au Moyen-Age européen, qu'aux Antilles. Le droit canon, par une décrétale célèbre d'Urbain III de 1187, accordait à de tels enfants la liberté. Mais ce sont les coutumes laïques, rétrogrades (Nivernais, Bourbonnais, etc.) qui appliquèrent le principe : le pire emporte le bon, principe on ne peut plus favorable au patrimoine seigneurial. En Bourgogne, inversement, on se réfère à la condition paternelle. Une affaire célèbre en Martinique, l'aff. Jean Boury, illustre indirectement ce contentieux, s'agissant d'une union passagère d'un libre affranchi et marié et d'une serve (Cf. arr. Cons. Souv., 2 janvier 1698, rendu entre J. Boury et les religieux tenant l'hôpital de l'île). L'enfant relève de la condition maternelle en raison de l'art. 6, al. 2, 3, 4 et 5 de l'Edit de mars 1685 . En l'espèce, ce n'est pas la turbatio sanguinis que l'on a voulu empêcher, mais le vice amoral de concubinage. V. notre étude sur la « Cura Morum aux Antilles », $R J P I C, 4^{\text {ème }}$ trim., pp. 320 et ss.
} 
l'ouest, la solution paraît résider dans le métissage. On a la volonté d'accroître la population par tous les moyens. Mais les métisses ne relèvent pas toujours, comme le révèlent les chroniqueurs, de la condition servile. On sait que les recensements de 1664 et de 1680 les situent avec les Blancs, surtout en Martinique. En Guadeloupe, compte tenu des difficultés économiques et financières propres à l'île, on aura tendance à ranger les mulâtres parmi les esclaves.

\section{LA QUESTION DISCIPLINAIRE}

Il nous faut distinguer ici deux aspects principaux : premièrement, la discipline des esclaves qui relève de l'officium du maître; deuxièmement, la discipline des maîtres qui est exercée de façon très mesurée par les officiers royaux, voire le Secrétaire de la marine ou le Roi.

\section{Juridiction domestique ou police de l'esclave}

A Rome l'esclave est naturellement placé sous la dominica potestas du chef de famille ${ }^{88}$. L'esclave relève donc tout naturellement de la juridiction domestique. Certains maîtres sont distants car ils n'habitent pas sur place; d'autres sont sévères; enfin, quelques uns sont clairvoyants, respectent l'humanité de l'esclave et ses désirs. Mais ces données nous viennent de la littérature dont il faut parfois se méfier ${ }^{89}$. Bien des auteurs estiment que, dans sa majorité, la société romaine fut

${ }^{88}$ Sur l'attitude des jurisconsultes classiques à l'égard de l'esclavage, V. Florentinus, Institutes, L.IX (= Dig. 1, 5, 4) : « La liberté est la faculté naturelle qui permet à chacun de faire ce qu'il veut, si ce n'est pas une chose interdite par la force ou le droit. La servitude résulte d'une décision du droit des gens, en vertu de laquelle une personne, contrairement à l'ordre naturel, est soumise à la maîtrise d'une autre. Les esclaves sont appelés serui parce que les empereurs ont coutume de vendre les captifs au lieu de les tuer et par là de les conserver. On les appelle aussi mancipia parce qu'ils sont enlevés aux ennemis avec les mains ». S'opposent donc les notions de ius naturale, favorable à la liberté, et le ius gentium, sorte de droit international public et privé de l'Antiquité, favorable à l'institution esclavagiste.

${ }^{89}$ Il convient donc de se montrer prudent et de distinguer les esclaves qui relèvent de la familia urbana proches du maître, policés, parfois très instruits, et les autres, durement traités car ce sont des manœuvres ruraux employés dans les champs (familia rustica). 
sévère et rigide à l'égard des esclaves aussi bien pour des raisons de crainte, de peur ou d'orgueil. Sénèque va jusqu'à rappeler l'adage traditionnel «tot hostes, quot seruos ${ }^{90}$. L'hostilité à l'égard des esclaves transparaît dans les écrits de Tacite $^{91}$ et nous la retrouvons naturellement transposée dans le monde des Antilles. Il convient de donner un pouvoir absolu au maître pour contenir un si grand nombre $\mathrm{d}^{\text {'hommes }}{ }^{92}$. Dès le XVII ${ }^{\text {ème }}$ siècle, selon le Père du Tertre, « la justice ne prend point connoissance de ces sortes de fautes [le marronnage] mais en laisse le châtiment à la discrétion des maîtres. Il n'y a que la révolte qu'on punit inexorablement du dernier supplice ${ }^{93}{ }^{9}$. Le leitmotiv est toujours en fait le suivant : le pouvoir quasi absolu du maitre repose sur l'idée que ce dernier doit contenir par tous les moyens un aussi grand nombre d'individus. D'où la supériorité, voire «l'infrangibilité » du maître blanc sur ses esclaves ${ }^{94}$. L'autorité publique semble intervenir principalement lorsque l'ordre public est en jeu. Un adoucissement des mœurs semble se produire à la fin de l'Ancien Régime. On trouve des annonces voire des affiches ou advis divers paraissant dans les gazettes locales ${ }^{95}$. Certaines proposent à l'esclave marron de revenir chez un voisin, ou chez un notaire. Parfois, il y a des promesses de non châtiment ou même de vente du fugitif à un autre maître ${ }^{96}$.

\footnotetext{
${ }^{90}$ Cf. Ep. $47,5$.

91 Cf. Annales, XIV, 42 ; V. égal, Pline, Ep. III, 14. Juvénal met en garde ses lecteurs contre les propos mauvais ou déformés tenus par les esclaves (Sat., IX, pp. 120-123).

${ }^{92}$ CHAN, col. F3 71 (idée de la supériorité nécessaire du Blanc).

${ }^{93}$ Du Tertre, op. cit., p. 496.

${ }^{94} \mathrm{CHAN}$, col. F3 71 .

95 En ce sens: Avis du 14 septembre 1789: «Azor, âgé d'environ vingt ans, à M. Laplace, greffier en chef de la sénéchaussée, à la Basse-Terre : s'adresser à Me. Blondet, procureur à la sénéchaussée de Basse-Terre », Cf. Gazette de la Martinique en date du 22 janvier 1784 (ADG 4 Mi 21 R1) : « La negresse Balsami, Ibo, agee de dix-sept ans [...] est marronne depuis vingt mois [...]; ses allures sont près de l'habitation de M. de Fougères, a Ste-Anne, et dans le bourg; ceux qui la feront conduire à Me. Mercier, notaire au Moule, auront une moëde de recompense; si elle se rend, elle aura sa grace ; on la vendra mêsmes, si elle a trouve un mestre ».

${ }^{96}$ Debien (G.), art. cit., pp. 17-18, met en avant la pratique de conciliation : dans ce cas le fugitif implorait l'assistance d'un voisin pour être conduit sur la plantation sous sa
} 


\section{Intervention de la justice publique}

Dans les cas de révolte armée, d'empoisonnements en série voire de soulèvement massif, la justice publique se doit d'intervenir. Jadis, à Rome, la question fut parfois posée notamment lors de révoltes sociales $^{97}$. Plus tardivement, au premier siècle, au Sénat, à la suite de l'assassinat de Pedanius Secundus (circa 61 de n.è.), un débat fut engagé sur l'opportunité de maintenir l'usage traditionnel qui consistait à mettre à mort tous les compagnons de servitude de l'assassin. Pline le Jeune, en tant que bon témoin de son époque, nous rapporte qu'un certain Larcius Macedo, renommé de triste mémoire, sénateur prétorien de son état - et lui-même fils d'affranchi-, fut tué par ses esclaves. Cet auteur poursuit en précisant au sujet de Macedo: "C'était d'ailleurs un maître orgueilleux et cruel, dont le père avait été esclave et qui l'avait trop oublié - à moins qu'il s'en souvint trop ${ }^{98}$. On retrouve parfois de tels cas d'espèce. Notamment dans l'affaire Dugez, un économe, l'instruction a permis d'établir que ce dernier avait bel et bien été massacré par les esclaves de l'habitation de Brinon dirigée par le Sr. La Sègue. Il y eut une dizaine de condamnations à la roue, une pendaison, des peines de fouet - notamment pour une femme ${ }^{99}$. On notera au passage que les maîtres, trop souvent absents de leur habitation, abandonnent toute autorité à un mandataire «irresponsable». Autre cas de figure: des

protection. Les colons pratiquaient entre eux ce genre de service d'ami, à la romaine. Mais il est évident que le colon intercesseur n'intervenait que lorsqu'il y avait des chances de pardon. De Ruggiero (V.), «Le marronnage en Guadeloupe à la veille de la Révolution française de 1789 »,BSHG, $\mathrm{n}^{\circ} 116-118,1998, \mathrm{pp}$. 5-64.

97 Gage (J.), Les classes sociales sous l'Empire romain, Paris, Payot, 1964 ; Alföldy (G.)Römische Sozialgeschichte, Wiesbaden, 1975. Auguste, en vertu de sa cura legum morumque se montra implacable à l'égard des esclaves : il prétendait que ceux-ci « souillaient la pureté du sang romain ». L'esclavage à Rome, contrairement aux idées reçues, est teinté de racisme.

${ }^{98} \mathrm{Cf}$. Ep. III, $14-1$ et 2.

99 Précision du procès mené contre les nègres du Marquis de Sennectère: CHAN, col. F3 226, pp. 133 et ss. 
affranchis possesseurs d'esclaves se montrent parfois eux aussi cruels, parfois vicieux ${ }^{100}$.

E. Petit, dans son ouvrage sur Le droit public des esclaves, rapproche les situations des esclaves des Antilles de ceux de l'Antiquité romaine. Il faut limiter les affranchissements et les réserver aux bons sujets, à ceux qui ont rendu des services exceptionnels tels que sauver les enfants du maître, protéger la maison, dénoncer les comploteurs. Ceux aussi qui ont découvert un poison inconnu avec indication des coupables ou qui fournissent des preuves ${ }^{101}$. On se souvient d'ailleurs qu'à Rome avait lieu une convention particulière, une offre ou pollicitatio qui se résume en une promesse de récompense à qui trouvera un esclave fugitif ${ }^{102}$. En revanche, la promesse de ramener un esclave mort ou vif à l'habitation est interdite par ordonnance des Administrateurs qui trouvent ce procédé très peu convenable ${ }^{103}$.

${ }^{100}$ Des gens de couleur libres sont parfois tuteurs (testamentaires) de Blancs (arr. Cons. Sup. du Cap, 14 octobre 1726); parfois ils sont à la tête d'une véritable fortune par suite de donations indirectes ou de rentes viagères: Cf. aff. Dausseur, arr. Cons. Sup. Guadeloupe confirmé par arrêt pris en Conseil du Roi le 23 avril 1774 - contra : aff. Lussy, arrêt Cons. Sup. Mart. (= Dessales, op. cit., p. 537) la Cour déclare : «Vouloir eviter que les negres ou mulastres libres ne devinssent trop puissants et que peu a peu les familles des Blancs ne cessent de s'appauvrir par ces donations ». On dispose aussi de l'affaire concernant la mulâtresse Allègre qui n'envoyait pas ses nègres participer aux offices. Plusieurs mulâtres, en effet, possèdent au XVII ${ }^{\mathrm{eme}}$ siècle des habitations (V. Abenon (L.-R.), th. cit., t. II, p. 151); plus récemment, on note un acquittement scandaleux d'Amé Noël, homme de couleur, âgé de soixante-douze ans lors de son procès, membre du Conseil privé du Gouverneur, qui torturait ses propres esclaves (C. Ass. Basse-Terre, 25 août 1839).

${ }^{101}$ Dr. pub. esc., p. 303.

${ }^{102}$ Sur l'incidium qui est une sorte de «pacte unilatéral », Giffard (A.-E.) et Villers (R.), Droit romain et ancien droit français, $4^{\text {eme }}$ éd., 1976, p. 184.

${ }^{103}$ Chauleau (L.) rapporte qu' $\ll$ un habitant ayant fait courir des billets par lesquels il promettoit une somme d'argent a qui luy ameneroit morte ou vie une de ses esclaves marronne [...] je [l'administrateur] l'ay faict mettre au fort ou il a (sic) reste un mois [...] personne n'estant en droict de mettre a prix la tete de qui que ce soit » (op. cit., p. 161). 
La police des maîtres est naturellement très difficile à mettre en cuvre dans un système esclavagiste où l'autorité du maître se doit de paraître indiscutable et ses décisions infaillibles, voire irrévocables. La conception du temps est toujours la même : il faut un «pouvoir absolu du maître pour contenir un si grand nombre d'hommes $»{ }^{104}$. Parmi les mesures juridiques favorables à l'esclave on peut citer la loi Petronia de 19 ap. J.-C. : le magistrat a la faculté de contrôler la décision du maître de livrer ses esclaves aux bêtes ${ }^{105}$. On l'a vu plus haut, un Edit bien connu de Claude prive de son droit de propriété le maître qui abandonne son esclave lorsqu'il est âgé et malade ${ }^{106}$. Enfin, la pression étatique se fait davantage sentir lorsque l'Empereur Antonin menace de peines le maître qui tue son esclave sine causa ${ }^{107}$. On peut dès lors penser que la mise à mort n'est autorisée que s'il y a un juste motif. Toutes ces anciennes solutions ont à notre avis profondément influencé les auteurs du Code Noir.

L'article 27 du Code précise que les esclaves infirmes «par vieillesse, maladie ou autrement, soit que la maladie soit incurable ou non, seront nourris et entretenus par leurs maîtres, et en cas qu'ils les eussent abandonnes, lesd. esclaves seront adjuges a l'hospital, auquel les maîtres seront condamnés a payer six sols par chascun jour pour la nourriture et entretien de chascun esclave $"$.

On dénombre divers cas de sévices sur les esclaves. On a vu plus haut que le droit romain classique ( $\mathrm{I}^{\mathrm{er}}-\mathrm{II}^{\mathrm{e} m e} \mathrm{~s}$.) avait essayé d'établir des mesures de contrôle en limitant la toute puissance voire le sadisme des maîtres et parfois des maîtresses ${ }^{108}$. Les cas d'excès et traitements inhumains abondent ${ }^{109}$ et ont été largement décrits par la doctrine. La psychose de l'empoisonnement fait que certains maîtres aux nerfs fatigués ou psychopathes obtiennent des aveux provoqués par les tortures

\footnotetext{
${ }^{104}$ Sur l'idée de la supériorité nécessaire du Blanc, CHAN, col. F3 71.

${ }^{105}$ Dig., 48, 8, $11-2$.

${ }^{106}$ C. J., 7, 6, 1, 3; Suétone, Claudius, XXV, 4.

${ }^{107}$ V. Gaius, op. cit., I, 53.

${ }^{108}$ V. Juvénal, Sat. XIV, 26 ; I, 92 ; VI, 475 ; XIV, 17.

${ }^{109}$ E. gr. arr., 4 novembre 1743 (aff. Langlois), CHAN, col. F3 226, 65.
} 
les plus cruelles. A. Gauthier rappelle le cas d'un colon, Lejeune, qui se plaint que son père ait perdu cinquante-deux esclaves en six mois. «Il fait périr par représailles quatre noirs et deux noires mis à la question ${ }^{110}$. Fouchard précise qu' « on leur brûlait les pieds, les jambes et les cuisses et on leur mettait un bâillon que l'on ôtait de temps en temps ${ }^{111}$. C'est surtout à partir de 1726 puis 1750 que ce genre d'accusations se multiplie ${ }^{112}$.

Le pouvoir central de Versailles est conscient de l'existence de ces abus. Ne voit-on pas, par exemple, un ecclésiastique, l'abbé Raynal, dénoncer ouvertement la tyrannie des maîtres et l'utilisation excessive du fouet $^{113}$. Aussi, en plein siècle des Lumières, le Secrétaire d'Etat à la marine expédie une missive au sujet d'une sentence infligée à un maître criminel estimée trop légère: "Nous ne ferons aucune mention ici des considérations d'humanité qui s'élèvent en faveur des esclaves. Vous connoisses tout le malheur de leur condition, quelque soin que l'on prenne d'en cacher une partie aux administrateurs, et nous savons combien vous voudries pouvoir alleger l'infortune de cette classe d'hommes. C'est dans les mesmes vuës que nous vous demandons de ne pas laisser entièrement impunis les forfaits de Lejeune. Nous joignons ici

${ }^{110}$ Gauthier (A.), Les sæurs de solitude. La condition féminine aux Antilles du XVIIme au XVII 'eme siècle, Paris, éd. Caribéennes, 1985, p. 224 (sur l'idée obsessionnelle de l'obtention de l'aveu, reine des preuves). En métropole, il faut attendre deux édits de Louis XVI pour voir supprimer les questions préparatoires et préalables par une Décl. Du 24 août 1780 et un Edit du 8 mai 1788. V. Schapper (B.), «La diffusion en France des nouvelles conceptions pénales dans la dernière décennie de l'Ancien Régime ", in Voies nouvelles en histoire du droit, $X V I^{\text {ème }}$-XIX $X^{\text {eme }}$ siècles, Bibl. Fac. droit et sc. soc., Poitiers, PUF, 1991.

${ }^{111}$ Fouchard, ouvr. cit., pp. 333-334.

112 Debbasch(Y.), «Le crime d'empoisonnement aux îles pendant la période esclavagiste », Revue d'histoire française de l'outre-mer, t. L1, 1963, pp. 10-188.

113 Histoire philosophique et politique des établissements et du commerce des Européens dans les îles, Paris, 1783, p. 80. L'auteur, d'ailleurs, prophétise la vengeance légitime du peuple noir. «Il ne manque, dit-il, aux negres qu'un chef assez courageux pour les conduire a la vengeance et au carnage. Ou est-il le grand homme que la nature doit peut-etre [engendrer] pour l'honeur de l'espece humaine? Ou est-il ce Spartacus nouveau? » (Liv. XI, ch. 21). 
une expedition [...] de la procedure [...]. Du moins nous paroît-il important qu'elle déclare le Sr. Nicolas Lejeune incapable de posseder des nesgres a l'avenir et qu'elle vous ordonne de l'envoyer aussitôt en France s'il osoît reparoitre sur l'habitation de son pere, ou mesme dans cette colonie. Il nous parô̂t qu'il seoit utile que nous donnions de la publicite a ces ordres, afin de contrebalancer une partie des maux qu'a pu causer l'arrest qui a innocente Lejeune ${ }^{114}$.

$*$

$*$

*

Il est difficile de rester neutre lorsque l'on aborde le sujet de l'esclavage qui était et sera toujours une honte pour l'humanité. Faut-il, comme l'exprime si bien le Pr. J.-M. Breton, «s'agissant de l'appréhension d'un phénomène par essence "déraisonnable" $[\ldots]$ ne pas sombrer, comme d'aucuns "en mal d'imprécations en forme de pseudo catharsis éthique" [...] savoir raison garder ${ }^{115}$. De plus, il faut tenir compte de la mentalité des populations des XVII ${ }^{\text {ème }}$ et XVIII ${ }^{\text {ème }}$ siècles et se souvenir que la rigueur du droit pénal était souvent atroce en métropole comme dans les colonies.

La matrice romaine de l'esclavage ne fait aucun doute : plus de quarante dispositions, selon $\mathrm{P}$. Jaubert, semblent rapprocher l'Edit de mars 1685 à des éléments divers de la législation romaine. L'esclave est considéré comme une chose, une res en vertu du Code et d'un acte de notoriété rendu par le lieutenant civil du Châtelet de Paris ${ }^{116}$. Mais là, repose selon nous une grave méprise. En fait, nous savons que l'esclave

\footnotetext{
${ }^{114}$ Longue aff. Déroulée à Saint-Domingue, décrite dans AN col. F3, 90, pp. 258-268 ; V. missive du 23 décembre 1785.

${ }^{115}$ Breton (J.-M.), « Du Code Noir à la pérennisation du statut servile : l'exemple des Antilles françaises et de la Guadeloupe ", communication à l'Académie des sciences d'outre-mer (Paris), 22 octobre 1998. V. version abrégée parue à la RJPIC (fin 1998), pp. 165-187.

${ }^{116}$ Acte du 13 novembre 1705.
} 
est souvent compris comme un immeuble par destination, pars fundi ${ }^{117}$. Il serait bien plus juste, pour conserver la véritable philosophie romaine, d'affirmer que l'esclave est un élément de la familia du maître, et qu'à ce titre, les colons et les maîtres jouissaient d'un mancipium ou d'une potestas sur la personne de l'esclave. Ceci ne peut que s'expliquer par une distinction qui n'a plus cours à la fin de l'Ancien Régime : l'homme en servitude doit précisément sa condition dans le fait qu'il est encore considéré inconsciemment comme une res mancipi, comme l'humble prolongement de la personne du maître.

L'esclave devient personne juridique selon le Code Noir s'il est baptisé. Il peut se marier. Enfin le Code fut très mal appliqué. Les décisions des Conseils supérieurs et des Administrateurs essaient vainement, à contre-courant, de limiter les affranchissements jugés trop nombreux, de combattre le laisser-aller des maîtres, d'interdire les réunions, les danses et l'alcoolisme du désespoir ${ }^{18}$. Finalement, on sent que dès la fin du XVIII ${ }^{\text {ème }}$ siècle l'institution esclavagiste est condamnée et remise en question par les tenants des Lumières ${ }^{119}$. Le Comité de législation hésite. Mais des propositions de suppression de l'esclavage conduiront à élaborer l'ultime projet - avorté pour fait de Révolution c'est-à-dire l'Edit sur la servitude de Louis $\mathrm{XVI}^{120}$, qui lui tenait à cœur.

117 Géraud-Llorca (E.), «La coutume de Paris outre-mer », Revue d'histoire du droit, vol. 60,1982 , p. 247.

118 Cf. CG, Ord. Des Administrateurs, 6 février 1691, CHAN, col. F3 221, p. 807, arr. Cons. Sup., 30 décembre 1691 interdisant la vente en détail des eaux-de-vie de canne ou devant leur porte [...] aux nègres « en sorte que lesd. negres s'enivrent et se gastent de boisson et ensuite se querellent ».

${ }^{119}$ On connaît peu de chose sur le rôle des loges maçonniques aux Antilles (E.g. : Loge de Saint-Jean de Jérusalem, de rite écossais au Cap). Il existe aussi des cercles de Philadelphes d'après Moreau de Saint-Méry. Curieusement, on se souvient que court une rumeur d'annonce de liberté générale en 1775, ce qui provoque la multiplication des Mémoires contre les affranchissements (AD Gir. C. 4383) «pour contrebalancer la propagande des prêcheurs d'émancipation $»$.

${ }_{120}$ Cf. Projet de loi sur le traitement et la police des negres serfs dans les colonies, élaboré en 1789, CHAN, col. F3 90, pp. 275 et ss. : "Art. $1^{\mathrm{er}}$. Nous abolissons a l'egard 


\section{RESUME}

De nombreux auteurs ont fait allusion au droit romain pour expliquer la logique - si tant est qu'il $\mathrm{y}$ ait une logique - du fameux Code Noir de 1685. A vrai dire, il conviendrait de se reporter non seulement aux travaux préparatoires, voire à l'expérience française du servage issue de la féodalité, mais encore, à notre avis, il paraît nécessaire de suivre l'application et l'évolution de l'institution servile au XVII $^{\text {ème }}$ et surtout au XVIII ${ }^{\text {ème }}$ siècle. En quelque sorte, la question principale est de savoir si la triste expérience de la servitude antillaise correspond à l'esclavage de l'Antiquité classique romaine ou encore à celui de l'Antiquité tardive $\left(I V^{\text {ème }}-V^{\text {ème }}\right.$ siècles, ou ère de la Romanité). Nous exposerons cette problématique en deux points. Après avoir retracé l'évolution et la flexibilité du modèle romain à la fin de l'Antiquité, le premier point portera essentiellement sur le phénomène de résurgence de la servitude gréco-romaine à la fin du Grand Siècle; le second, outre les parallélismes de forme ou de fond, s'attachera à dégager les signes communs d'évolution entre le "modèle» des Anciens et l'application des Modernes.

\begin{abstract}
Many scholars used to refer to Roman Law to explain the rationale - if any - of the well-known 1685 Code Noir. As a matter of fact, it would be useful to look back not only to preliminary works on that Code but also, in our opinion, to draw back the enforcement an evolution of slavery law in the XVIIth and, most of all, $X V I I I t h$ centuries. In some ways, the main point is to know whether slavery in the Caribbean was looking like slavery at Rome in classic or late period (i.e. from IVth to VIIth century, so-called Romanity Era). We intend to disscuss this issue in two points. After looking back to the evolution and flexibility of Roman pattern at the end of Antiquity, our first point will mainly deal with the revival of Greek and roman-type slavery at the end of the Great Century; our second point will discuss patterns of evolution of slavery that are common to both oldtype and modern slavery.
\end{abstract}

des negres [...] la qualification d'esclaves, comme presentant une dependance illimitee ». 